\title{
P I 0-05. Suppression of human dendritic cell function during acute HIV infection
}

\author{
D Frleta*1, M O'Brien ${ }^{1}$, B Haynes ${ }^{2}$, B Kessler ${ }^{3}$ and N Bhardwaj ${ }^{1}$
}

\author{
Address: ${ }^{1}$ Cancer Institute, New York University Langone Medical Center, New York, USA, ${ }^{2}$ Duke University Medical Center, Durham, NC, USA \\ and ${ }^{3}$ Oxford University, Oxford, UK \\ * Corresponding author
}

from AIDS Vaccine 2009

Paris, France. 19-22 October 2009

Published: 22 October 2009

Retrovirology 2009, 6(Suppl 3):PI36 doi:I0.I I86/I742-4690-6-S3-PI36

This abstract is available from: http://www.retrovirology.com/content/6/S3/PI36

(C) 2009 Frleta et al; licensee BioMed Central Ltd.

\section{Background}

During acute HIV infection (AHI), CD4+ T cells undergo apoptosis from direct infection and through bystander apoptosis induced by inflammatory factors. Both the inflammatory factors and apoptotic material produced during AHI may negatively impact innate and adaptive immune responses. Such inhibitory factors present in AHI plasma include apoptotic microparticles (MPs), small membranous blebs derived from dying cells. Dendritic cells (DCs) are the sentinel cells of the immune system that respond to pathogens, frequently through Toll-like receptor (TLR) recognition of unique pathogenic macromolecules. Because TLR recognition of viral material by DCs is important for DC priming of virus-specific T cells, we determined how AHI patient plasma or apoptotic MPs affect TLR-stimulated DCs.

\section{Methods}

Plasma was obtained from acute HIV patients or healthy donor controls. Apoptotic MPs were isolated from supernatant of UV-irradiated or HIV-infected PBMCs. Human DCs were treated with MPs or $10 \%$ plasma (control or AHI) and subsequently stimulated with various TLR agonists. DC activation was then assessed by examining DC phenotype, cytokine production, and ability to activate naïve allogeneic or autologous memory CD4+ T cells. MPspecific receptors were isolated from the DC surface and sequenced by mass spectrometry.

\section{Results}

AHI plasma, relative to healthy plasma, inhibited TLRstimulated DC cytokine production. Because apoptotic MPs are present at high levels in AHI plasma, we treated DCs with MPs and observed reduced DC activation. The inhibition of DCs by AHI or MPs blocked DC capacity to induce IFNgamma-producing T helper $1 \mathrm{CD} 4+\mathrm{T}$ cells, which are critical regulators of cell-mediated immunity. MP-specific DC receptors include various integrins, CD97, HLA class I, and voltage-dependent anionic channels (VDAC).

\section{Conclusion}

Determining the factors in AHI, such as apoptotic MPs, which block DC function will provide potential targets to stimulate HIV-specific immunity. We are currently investigating molecular mechanisms of AHI and MP-mediated DC inhibition. 